\title{
Selbstoptimierung und Enhancement
}

\section{Der sich verbessernde Mensch - ein expandierendes Forschungsfeld}

\author{
Oswald Balandis \& Jürgen Straub
}

Journal für Psychologie, 26(1), 131-155

https://doi.org/10.30820/8247.09

www.journal-fuer-psychologie.de

\section{Zusammenfassung}

Das Bemühen, sich und seine Welt zu verbessern, ist anthropologisch-universal. Gleichwohl sind bestimmte Praxen der (unentwegten) Selbstoptimierung und des Enhancements historische und soziokulturelle Besonderheiten, die ebenso besondere Subjekte mit spezifischen Begehren, Bedürfnissen und Bemühungen hervorbringen. Der Übersichtsbeitrag eröffnet nach detaillierten Begriffsbestimmungen einige kritische Einsichten in exemplarische Praxis- und Forschungsfelder, in denen die (teils technisch vermittelte) Optimierung des Selbst im Zentrum steht. Dazu zählen Ernährung, Fitness und Gesundheit, Schönheit, Sexualität und Schlaf, alle möglichen physischen und kognitiven Leistungen, emotionale Fähigkeiten und soziale Kompetenzen (die in psychotherapeutischen beziehungsweise psychotechnischen Behandlungen sowie verwandten Beratungen, Trainings und Coachings fokussiert werden). Das zeitgenössische Optimierungsbegehren begnügt sich nicht mit bloßen Verbesserungen des Althergebrachten. In trans- oder posthumanistischer Zeit tritt es transgressiv auf und zielt - sei es in technomanischen Entwürfen, sei es in feministischer Perspektive - auf die Cyborgisierung des menschlichen Organismus und die Abschaffung des » antiquierten Menschen «. Selbstoptimierungen reichen mithin von traditionellen Selbstformungen und psychotechnischen Manipulationen über pharmakologische und chirurgische Modifikationen sowie prothetische Ergänzungen und Erweiterungen bis zur gehirnphysiologisch installierten Steuerung des Menschen und schließlich seiner genetischen Transformation in einer $>$ positiven Eugenik $<$ und $>$ neuen Evolution $<$.

Schlüsselwörter: Optimierung, Enhancement, Selbst, Körper, Cyborg

\section{Summary}

The Self-Improving Human. Optimization and Enhancement in Socio-scientific Research and Cultural Studies

Attempts to improve oneself and the world are anthropologically universal. At the same time, certain practices of (continuous) self-optimization and enhancement are historical and so- 
cio-cultural particularities which produce particular subjects with particular desires, needs, and efforts. After defining certain terms, this article offers a critical overview and insights into exemplary research and practice fields which center around the (sometimes technologically mediated) self-optimization. Examples are diets, fitness, health, beauty, sexuality, sleep, all kinds of physiological and cognitive achievements, emotional abilities and social competencies (which are focused on in psychotherapeutic and psycho-technological therapies and related consultations, trainings, and coachings). The contemporary desire for optimization is not satisfied with a simple improvement of the traditional. In trans- and posthuman times, it is transgressive - be it in techno-manic sketches, in a feminist perspective - and aims at a cyborgization of the human body and the end of the antiquated human. Self-optimization reaches from traditional creations of the self to psychotechnological manipulation, pharmacological and chirurgic modification and prosthetic additions/extensions all the way to brain-physiological implementations of control of human beings and eventually genetic transformations in a > positive eugenics $<$ and $>$ new evolution $<$.

Keywords: optimization, enhancement, self, body, cyborg

\section{Optimierung: anthropologische, gesellschaftliche und psychosoziale Bedeutung}

Der Mensch ist das sich selbst und seine Welt verbessernde Lebewesen (Assmann \& Assmann, 2010; Kipke, 2011; Sloterdijk, 2009). Diese anthropologische Bestimmung schließt andere nicht aus, auch solche nicht, die die Destruktivität menschlichen Handelns hervorheben. Mitunter münden Verbesserungsversuche sogar in zerstörerische Ergebnisse und Folgen. Im Hinblick auf die hier interessierende anthropologische Konstante lässt sich formulieren: Das allgemeine Ziel der Optimierung ist tief in die Grundstruktur intentionalen Handelns eingelassen. Menschliches Handeln mag häufig um die Erhaltung eines Status Quo bemüht, also bewahrend oder konservativ sein. Ebenso oft bezweckt es Veränderungen (Modifikationen, Innovationen) und begreift diese als Verbesserungen. Optimierungsabsichten schützen freilich weder vor dem Scheitern noch vor nicht intendierten, negativen Folgen und Nebenfolgen intentionalen Handelns. Im Übrigen teilt auch das um Verbesserungen besorgte Handeln ein Charakteristikum allen Tuns und Lassens: es ist überdeterminiert und polyvalent, kann also stets von mehreren - widersprüchlichen, sich widerstreitenden, auch unbewussten - Motiven gespeist sein und vielfältige Bedeutungen besitzen. $O b$ optimierende Handlungen (ausschließlich) ihrem bewusst gesetzten, rational bedachten und planvoll verfolgten Zweck dienen, ist grundsätzlich nur im Nachhinein zu beurteilen, manchmal erst lange Zeit nach dem Vollzug der betreffenden Handlungen. Was der Verbesserung 
dienen sollte (etwa die Einnahme eines leistungssteigernden Medikaments), erweist sich ex post festum mitunter sowohl in seiner Motivationsstruktur als auch in seinen Folgen und Nebenfolgen als schädigend.

Wer optimierendes Handeln als anthropologische Universalie auffasst, muss sogleich historische, kulturelle und gesellschaftliche Relationierungen sowie interindividuelle Differenzierungen geltend machen. Nicht immer und überall begegnen uns Menschen als in gleicher Weise und mit derselben Intensität an Optimierungen (oder sogar an der Vervollkommnung) ihres Selbst und ihrer Welt interessierte sowie ihr Handeln dauerhaft darauf abstellende Akteure (z. B. Assmann \& Assmann, 2010; King et al., 2017; Mayer et al., 2013). Erhebliche Variationen auf kollektiver und individueller Ebene sind evident. Der Wille zur Optimierung sowie die zu seiner Verwirklichung erforderliche praktische Haltung, der passende soziale Habitus und eine förderliche psychische Disposition sind kontingente Phänomene. Sie sind in einzelnen Gesellschaften und Gemeinschaften ebenso unterschiedlich ausgeprägt wie das für Optimierungen notwendige Wissen und Können sowie hilfreiche Technologien und Techniken. Historisch-soziologische und psychohistorische, zeitdiagnostisch gehaltvolle Untersuchungen sind sich darin einig, dass sich die » heißen «, hoch komplexen und extrem dynamischen, beschleunigten Gesellschaften (besonders) seit dem 20. Jahrhundert zunehmend dadurch auszeichnen, dass kontinuierlich erneuerte, radikalisierte und erweiterte Optimierungsbegehren sowie ihre unablässige Realisierung alle gesellschaftlichen Subsysteme sowie die Lebenswelt zunehmend durchdringen und prägen. Allerlei Optimierungsprogramme und -projekte bilden gegenwärtig einen Grundzug > spät<- oder > postmoderner < Lebensformen. Das zeigt sich längst auch an den Individuen selbst, an ihrer psychischen Verfassung und persönlichen Lebensführung (King, 2013a; King et al., 2014; King \& Gerisch, 2015).

Zahllose Freuden und Leiden von Menschen haben mit gelungenen beziehungsweise gescheiterten Optimierungsversuchen zu tun, von denen abzulassen bereits als (negativ sanktionierter) Verstoß gegen anerkannte Werte und verbindliche Normen gilt. Die allgegenwärtigen Appelle zum lebenslangen Lernen (Meyer-Drawe, 2008) belegen dies ebenso wie die unentwegten Aufforderungen zur Entwicklung und Steigerung von allerlei Kompetenzen (Reichenbach, 2009; Straub, 2017). In vielen Gesellschaften kann sich heute kaum noch jemand dem Einfluss des generalisierten Imperativs eines hegemonial gewordenen Optimierungsregimes gänzlich entziehen: »Du musst Dein Leben ändern! «, zitiert Sloterdijk (2009) im Titel seines Buches über Anthropotechnik den Dichter Rainer Maria Rilke. Gemeint ist mit diesem Appell freilich nicht irgendeine Veränderung, sondern eine Optimierung des Selbst und der Welt des Menschen. Die allerorts und andauernd präsente Aufforderung ist an alle adressiert. Sie ist in allerlei kommerzielle Kontexte eingebettet. Sie hält die Angerufenen an, Verantwortung für sich und die ständige Selbstoptimierung zu übernehmen (ganz im Sinne eines unternehmerischen Selbst, Bröckling, 2007). Sie ist Bestandteil einer viele Le- 
bensbereiche und Handlungsfelder durchdringenden, machtvollen Bio- und PsychoPolitik (Foucault, 2006; Gehring, 2006; Rose, 1999, 2010; Volkers \& Lemke, 2014). Sie verspricht Leistungssteigerungen aller Art und Wohlbefinden, Erfolg und Glück. Sie betrifft Menschen jeden Alters, jeder sozialen Herkunft und kulturellen Zugehörigkeit, jeden Geschlechts. Alle sollen sich angesprochen fühlen, wenngleich nicht immer in ein und derselben Weise. Insbesondere geschlechtsspezifische Differenzierungen sind auch hier zu finden. Man denke etwa an das der Selbstoptimierung dienende Gesundheits- oder Schönheitshandeln (siehe unten). Im Folgenden geht es ausschließlich um Selbstoptimierungen (und das Human Enhancement als eine besondere Variante dieser Praxis), zunächst um eine definitorische Bestimmung, die auf einer Analyse der Pragma-Semantik der interessierenden Begriffe beruht.

\section{Optimierung, Enhancement: Begriffsklärung}

$\gg$ Optimieren «, »Optimierung «, »Optimum «, »optimal « und verwandte Terme stammen aus dem Lateinischen (optimus, -a, -um: der, die, das Beste, Tauglichste, Tüchtigste usw.). Sie haben ihre wesentlichen Bedeutungen im Deutschen und anderen Sprachen bis heute bewahrt. Als grundsätzlich äquivalent gelten Wörter wie »verbessern «, oder »Verbesserung «. Stets geht es mit diesen auf Tätigkeiten und deren Ergebnisse bezogenen Wörtern um eine Art Steigerungslogik sowie das Anstreben und Erreichen eines - bis auf weiteres und vergleichsweise - besten Zustandes. Die Vorläufigkeit des relativ Besten trennt die Optimierung von der Perfektionierung, also das unter den gegebenen Umständen Optimale vom Perfekten oder Vollkommenen. Terminologisch lassen sich die (pragma-semantisch) verwandten Begriffe gut unterscheiden (obwohl sie häufig gleichbedeutend gebraucht werden und austauschbar erscheinen). Folgende Prädikatorenregel ist hilfreich: Während die Perfektionierung auf einen absoluten, nicht mehr verbesserungsbedürftigen und -fähigen Zustand abzielt, hat die Optimierung kein endgültiges Ende, nichts Definitives vor Augen. Beide Tätigkeitswörter (optimieren, perfektionieren; verbessern, vervollkommnen/vollenden) bezeichnen innovative oder transformative Praxen. Allein die Optimierung ist wegen ihrer pragmasemantisch vorausgesetzten Unabschließbarkeit ein Prozessbegriff par excellence. Optimierendes Handeln ist auf eine grundsätzlich offene Zukunft bezogen. Es bewegt sich auf einem Kontinuum ohne klar bestimmbares Ende. Sein Maßstab operiert nicht mit einer vorab festgelegten Grenze. Sein Ziel verschiebt sich häufig noch im Zuge optimierenden Handelns.

Dieser unabschließbare, auf immer neue Gegenstände ausdehnbare Prozess der aktiven Optimierung steht häufig im Zentrum wissenschaftlicher Aufmerksamkeit. Zuständig für diese (womöglich an technologisches Wissen sowie die Verwendung 
technischer Apparate, Instrumente, Verfahren und Produkte gekoppelten) Verbesserung sind dabei nicht nur Expert_innen. In Optimierungsgesellschaften und -kulturen sind neben einem Heer von dienstbaren Berater_innen und Therapeut_innen, Trainer_innen, Coachinnen und Coaches, die mit Anleitungen und Anweisungen zur Selbstoptimierung ihrer Klient_innen zur Hand sind, die Individuen selbst aufgefordert, sich beständig um sich zu kümmern. Sie sollen sich unentwegt beobachten und dem internalisierten Imperativ der Selbstverbesserung Folge leisten.

Die Praxis der Selbstoptimierung erfüllt mehrere Funktionen zugleich. Sie bringt Menschen, zumindest im Erfolgsfall, ihren persönlichen Zielen näher (steigert ihr Wohlbefinden, ihr Glück usw.). Im selben Moment sorgt sie für die Anpassung von Individuen, die in dynamischen, konkurrenzbasierten und leistungsorientierten Gesellschaften nur durch immerwährende Verbesserungen funktionstüchtig und chancenreich bleiben. Ihr Erfolg in kompetitiven Verhältnissen hängt von der unaufhörlichen Entwicklung von Kompetenzen ab. Die Pragma-Semantik von »Kompetenz « (»kompetent «) setzt Konkurrenz und Kompetition voraus (Straub, 2007). Allerlei Verbesserungen, mitunter aufwendige und mühsame Selbstoptimierungen dienen dem einer generellen Steigerungslogik unterworfenen und sich dem Wettbewerb stellenden Subjekt als instabile, stets zu erneuernde Grundlage des eigenen (Über-)Lebens und Fortkommens. Das sich in diesem Sinne anpassende Selbst erfüllt so auch eigene Bedürfnisse, persönliche Begehren und Wünsche. Sich selbst optimierende Subjekte erlangen in der Regel Aufmerksamkeit und Anerkennung durch andere, genießen neben der ausgeübten Selbstkontrolle und -wirksamkeit auch Erfolge und die ihnen womöglich zuteilwerdende Ehre. Wegen der (manchmal kaum mehr merklichen, überaus subtilen) Verschränkung von Heteronomie und Autonomie in Praxen der Selbstoptimierung kann man dieses Subjekt » auteronom « nennen (Straub, 2013). Solche Subjekte werden verführt und geführt und führen sich dennoch auch selbst (im vorgegebenen Rahmen). Sie verschränken und verweben in ihrem auf Optimierung abzielenden Handeln und Leben Fremdsteuerung und Selbstbestimmung.

Optimieren und Optimierung, verbessern und Verbesserung: Wir bezeichnen mit diesen Begriffen nun genauer ein Geschehen oder Vorgänge, meistens spezielle Tätigkeiten oder Handlungen, die zielgerichtete Veränderungen herbeiführen (sollen) und dabei an expliziten oder impliziten Kriterien orientiert sind. ${ }^{1}$ Wir gebrauchen die (substantivierbaren, allerlei Derivate mit sich führenden) Verben in unserer Alltags-, Bildungs- und Wissenschaftssprache als mindestens zweistellige Prädikatoren, sodass sich als erste Bestimmungsmerkmale ergeben:

1. Ein Subjekt (oder eine Instanz) optimiert etwas, also

2. irgendein Objekt (oder einen Gegenstand).

3. Im Fall der Selbstoptimierung fallen das Subjekt und das Objekt in eins, sodass man spezifizieren und weiter differenzieren kann: Personen vermögen sich be- 
ziehungsweise etwas an sich durch selbstbezügliche Handlungen im Sinne der erwünschten Verbesserung gezielt zu verändern (Analoges gilt für Kollektive. Das bleibt im Folgenden unberücksichtigt).

Weitere, den Gebrauch des Prädikators präzisierende Stellen können leicht ergänzt werden. In der Philosophie und den Wissenschaften sind solche Erweiterungen wegen der notwendigen Genauigkeit sprachlicher Verständigung unumgänglich. Folgende sind besonders wichtig:

1. Es lässt sich neben dem Subjekt und Objekt angeben, in welcher speziellen Hinsicht etwas - zum Beispiel das Verhalten, der Körper, die Leistungsfähigkeit oder die Persönlichkeit eines Menschen - ein Gegenstand von Optimierungsvorgängen ist (sein soll etc.).

2. Die Angabe einer solchen Hinsicht setzt ein (zum Beispiel technisches, medizinisches, psychologisches, pädagogisches, ästhetisches, ethisch-moralisches, religiöses) Wissen

3. sowie ein damit verbundenes (qualitatives oder quantitatives) Kriterium voraus, an dem sich bemessen lässt, was als (gelungene oder misslungene) Optimierung gelten kann. ${ }^{2}$

4. Außerdem ist anzugeben, für wen sich die Veränderung als Optimierung darstellt, wer also einen Nutzen aus ihr zieht.

5. Weiterhin ist interessant, wodurch sich die Optimierung vollzieht, was mithin geschieht beziehungsweise getan wird im Zeichen und mit dem intendierten Effekt einer sich einstellenden Verbesserung.

6. Ebenfalls ist relevant, wie und - falls man es nicht mit einem $>$ naturwüchsigen $<$, sich von selbst einstellenden Vorgang zu tun hat (zum Beispiel Wachstum, Reifung) - mit welchen Verfahren und Mitteln, Instrumenten, Apparaten oder Techniken die resultierende Verbesserung bewerkstelligt wird.

7. Schließlich mag man untersuchen beziehungsweise angeben, welche intendierten und welche unbeabsichtigten Folgen und Nebenfolgen das erreichte Ergebnis einer Optimierung tatsächlich zeitigt/bewirken kann (kurzfristig, mittelfristig, langfristig; reversibel oder irreversibel; positiv oder negativ für den oder die Betroffenen usw.).

Die angeführten Stellen verweisen aufeinander. Sie gehören allesamt zur $\gg$ Logik « beziehungsweise Semantik und Pragmatik des interessierenden Prädikators. Die vorgenommenen Unterscheidungen und Begriffsbestimmungen spezifizieren leitende Perspektiven und mögliche Akzentsetzungen, unter denen wissenschaftliche Analysen von Optimierungsvorgängen durchgeführt werden können (naturwissenschaftliche, etwa biologische oder medizinische, sowie geschichtswissenschaftliche oder sozial- und kul- 
turwissenschaftliche, einschließlich inter- und transdisziplinäre wie etwa in den Cultural Studies oder Gender Studies).

Häufig wird das Enhancement nicht vom Begriff der Selbstoptimierung unterschieden. Von Enhancement war zuerst vor allem in der Medizinethik die Rede (Juengst, 1998; Lenk, 2006). Der Begriff ist dort auf das Problem bezogen, therapeutisch-kurative Eingriffe von »verbessernden « (optimierenden, nicht-kurativen) abzugrenzen. Während erstere eine Erkrankung oder Störung voraussetzen, ist das bei pharmakologisch, neurologisch und biotechnologisch begründeten Verfahren des Enhancements nicht der Fall. Wichtig für die Pragma-Semantik des medizinischen beziehungsweise medizinethischen Begriffs ist außerdem die Tatsache, dass mit einem Enhancement irgendein Eingriff in die Integrität des Körpers verbunden ist. Einschlägige Maßnahmen beruhen also auf ärztlicher Kunst, medizinischem Wissen und technischem Können. Dieser Aspekt lässt sich für eine allgemeine Definition heranziehen. Unser Vorschlag entspricht durchaus geläufigen Unterscheidungen, die jedoch selten systematisch expliziert und konsequent beachtet werden: Enhancement ist eine Sonderform der Selbstoptimierung. Jedes Enhancement eines Selbst beziehungsweise Selbstaspekts ist eine Optimierung, jedoch nicht jede Selbstoptimierung ein Enhancement (obwohl die Grenzen fließend sein können, die getroffene Unterscheidung also akzentuierend angelegt ist). Zum Enhancement zählen alle naturwissenschaftlich fundierten und technologisch begründeten sowie (mehr oder weniger) technisch voraussetzungsvollen und apparativ aufwendigen Varianten der Selbstoptimierung. Entsprechende Maßnahmen sind von professioneller Anleitung und Unterstützung abhängig. Vielfach sind sie direkt an Dienstleistungen von Expert_innen gebunden (die zum Beispiel als plastische Chirurg_innen Gesichter modellieren oder als Neurolog_innen Maßnahmen des Neuroenhancement einleiten, die ohne naturwissenschaftliches Wissen und technisches Können nicht möglich wären). Im Grunde genommen besitzen viele biologisch und medizinisch fundierte Praxen der Selbstoptimierung - speziell des Körpers beziehungsweise irgendwelcher physischen oder physiologischen Aspekte paradigmatischen Status. Optimierende Interventionen - von medikamentösen Behandlungen über chirurgisch-invasive Maßnahmen und prothetische Ersetzungen oder Ergänzungen (Straub \& Métraux, 2017) bis hin zu modifizierenden Eingriffen in die genetische Ausstattung oder das Gehirn eines Menschen - erfüllen nicht ausdrücklich beziehungsweise vorrangig therapeutische Funktionen. Selbstoptimierungen reparieren oder heilen nicht nur, sondern steigern, überschreiten, erweitern und ersetzen Vorhandenes. Sie sind gerade als wissenschaftlich, technologisch und technisch aufwendiges Human Enhancement transgressiv und fügen dem Gegebenen und Gewohnten etwas Neues hinzu (vgl. - aus kritischer oder affirmativer Perspektive - z. B. Coenen et al., 2010; Gordijn \& Chadwick, 2008; Knoepffler \& Savulescu, 2009; Tirosh-Samuelson \& Mossman, 2012). 
Im Unterschied zu jenen traditionellen Formen der Sorge um sich, welche Kipke $(2011,2012)$ in der Kategorie der Selbstformung zusammenfasst (Straub, 2012a), sind Verfahren des Enhancements nicht an eigene, übende beziehungsweise trainierende Tätigkeiten des sich (oder etwas an sich) verbessernden Subjekts gebunden. Es macht einen Unterschied, ob man sich zu verbessern trachtet, indem man in mühsamen und langsamen Schritten eine Meditationstechnik erlernt und diese meditative Praxis dann regelmäßig ausübt, oder ob man eine Pille schluckt, um sich (in dieser oder jener Hinsicht) zu steigern (ohne vorher und währenddessen bestimmte Erfahrungen machen zu müssen). Die an kontinuierliche Selbstformung (Selbstgestaltung, Selbstbildung, Selbstentwicklung etc.) gekoppelte Optimierung der körperlichen Kondition (etwa beim täglichen Langstreckenlauf) ist etwas ganz anderes als das von Experten durchgeführte Enhancement, das eine hormonelle Umstellung oder den Eingriff in die Erbanlage oder in physiologische (Gehirn-)Strukturen einer Person vorsieht. Enhancements sind Selbstoptimierungen, die auf fremdes Wissen und Können angewiesen sind. Sie bestehen nicht oder kaum in Handlungen des optimierungswilligen Subjekts, sondern in Behandlungen durch andere. Sie bringen keine Erlebnisse und Erfahrungen mit sich, wie es selbstformende Tätigkeiten tun, sondern setzen auf (unmittelbare) Ergebnisse und Folgen. Wir gehen im Folgenden auf ausgewählte Beispiele aus verschiedenen Praxis- und Forschungsfeldern ein. Schon diese informative Übersicht verdeutlicht, wie sehr unsere Lebenswelt und viele Berufsfelder vom Gedanken der Optimierung des Selbst und seiner sozialen Beziehungen durchdrungen sind. Gerade auch die an technische Mittel gebundene Praxis des Enhancements ist einer Steigerungs- und Ausbreitungslogik verpflichtet, die keine Grenzen vorsieht und hinnimmt.

\section{Optimierende Handlungen und Behandlungen: Praxis- und Forschungsfelder}

Viele Verfahren und Praktiken der Selbstoptimierung greifen nicht nur in die Physis und Psyche der Einzelnen ein, sondern können auch soziale Beziehungen und soziokulturelle Lebensformen massiv verändern (worauf wir unten näher eingehen). Von oberflächlichen Änderungen bis hin zu radikalen Revolutionen überkommener Wirklichkeiten und überlieferter Möglichkeiten ist alles denkbar. Exemplarische Felder sind die folgenden: ${ }^{3}$

Der Blick auf den Umgang mit dem eigenen Körper eröffnet ein mannigfaltiges Interventionsfeld diverser optimierender Praktiken. In vielerlei Hinsicht wird der Körper »rohstoffisiert « (Villa, 2011, 2013) und somit ein Gegenstand, der be- und verarbeitet werden kann. Das mag dem Zweck dienen, ihn (kapitalistisch) verwertbar zu halten, gewissen ästhetischen Ansprüchen zu genügen oder die Funktions- und 
Leistungsfähigkeit im Sinne bestimmter biomedizinischer Kriterien zu gewährleisten (beziehungsweise zu steigern). Die (meta-)theoretische Basis für ein derartiges Verständnis des Körpers liefern die modernen Wissenschaften vom Menschen. Beispielhaft lässt sich dies anhand der Modifikation von Ernährungspraktiken zeigen, die niemals nur physiologische Notwendigkeiten erfüllen, sondern historisch, ökonomisch, gesellschaftlich, kulturell und sozial kodiert sind. Erst ab Mitte des 19. Jahrhunderts wird Ernährung als Energiezufuhr, Nahrung als Energieträger und der Körper als eine Art $\gg$ Verbrennungsmotor « konzipiert, welcher die eingeführte Energiemenge in kinetische Energie und Wärme umwandelt (Neswald, 2013). Diese »chemische Buchhaltung « von Kalorien entstand im Kontext des sozio-historischen Notstands, die aufkommende industrielle Produktionsweise mit einer ausreichenden Anzahl an Arbeitskräften zu versorgen, indem Fragen von Hunger und Ernährung nach rationalen Kriterien beurteilt und experimentell erforscht wurden. Physiologen, Hygieniker sowie Sozialreformer waren sich einig: Energiephysiologisch ist Essen einer der wichtigsten Aspekte der individuellen Reproduktion von Arbeitskraft. Man muss essen, um arbeiten zu können, und das möglichst kosten- und energieeffizient. Dazu bedarf es einer kalkulatorischen Grundlage der Optimierung (Ernährungswissen, Kalorientabellen etc.).

Obwohl Ernährung heutzutage für viele (insbesondere in westlichen Gesellschaften) keine derartig karge Angelegenheit mehr ist, bezeugen die unzähligen Beratungsangebote, Praktiken der Kaloriendokumentation und aktiven Gewichtskontrolle die Durchschlagskraft und Persistenz der ernährungsphysiologischen Buchhaltung. Mehr noch: in Zeiten von Übergewicht und Adipositas zeigt sich, dass Fragen der Gesundheit eines Körpers mit ästhetischen Beurteilungen einhergehen und mit einer zunehmenden, gesellschaftlich und kulturell vermittelten Eigenverantwortung für das eigene körperliche Wohl und ästhetische Glück verquickt werden (Schmidt-Semisch \& Schorb, 2008). Doch nicht nur das Dicksein wird als Abweichung und alleinige Konsequenz der eigenen Entscheidungen und Handlungen betrachtet, auch die Verantwortung für die Reduktion des Gewichts soll kraft eigener Anstrengung erfolgen. Permanente (Selbst-)Beobachtungen der (ernährungs- und bewegungsabhängigen) Körper-Form nach Maßgabe einer allgemeinen Fitness (mitunter im Zeichen sportlicher Leistungsfähigkeit) sind exemplarische Praktiken der Selbstdisziplinierung und Selbstoptimierung (Duttweiler, 2003, 2012).

Neben allgemeiner Fitness ist die körperliche Schönheit ein bevorzugtes Interventionsfeld optimierender Maßnahmen (in Beratungs- und Empfehlungsregimes). Es ist evident, dass geschlechtsspezifische Kodierungen einschlägiger Maßstäbe, Maßnahmen und Handlungsformen gerade in diesem Feld gängig sind. Zwar machen sich Menschen jedweden Geschlechts (bisweilen oder beständig) schön (nach variablen Kriterien). Doch sind es (bislang) insbesondere Frauenkörper, die in den Medien und der Le- 
benswelt besondere Aufmerksamkeit auf sich ziehen (Villa \& Zimmermann, 2013). Wer schön sein will, muss leiden und vor allem so einiges tun: »Schönheitshandeln « ist dabei nur vordergründig eine Frage individueller Präferenzen und Selbstermächtigung (»ich mach das für mich«). Vielmehr zeigt sich auch hier, dass Schönheit eine soziale und kulturelle, wirkmächtig normierte Angelegenheit ist, die mit Möglichkeiten der Erlangung beruflicher Positionen, gesellschaftlichem Status und sozialer Anerkennung einhergeht (Degele, 2004, 2008). Schönheit als Attraktivität (für andere) ist eine soziokulturelle, in Macht- und Herrschaftsbeziehungen (in patriarchalischen Geschlechterordnungen) eingebettete Angelegenheit und häufig als solche Objekt optimierenden Handelns.

Gegenwärtig stehen Myriaden chirurgischer Optionen und technischer Enhancement-Verfahren zur Verfügung, die - je nach finanziellem Aufwand und der Radikalität des Eingriffs in die körperliche Integrität - vielfache Modifizierungen des eigenen Körpers ermöglichen. Fast immer wird dabei medizinisches Fachwissen für nicht-therapeutische und nicht-reparative Zwecke mobilisiert, um den Körper oder einzelne Körperpartien modellieren zu lassen (vgl. z. B. Ach, 2006; Borkenhagen et al., 2016). Zwar werden schönheitschirurgische Maßnahmen gesellschaftlich durchaus als Vehikel für eine »Democratization of Beauty « (Rosen, 2004) diskutiert. Dies geschieht jedoch häufig unter Ausblendung aufzubringender Mühen (nicht nur in Form von Schmerzen und, bei misslungenen Eingriffen, von Entstellungen) sowie der damit einhergehenden individuellen Verantwortung für körperliche Schönheit. Als (vermeintlich) vollkommen freie Entscheidung souveräner Individuen aufgefasst, tritt die biopolitische Dimension dieser Praktiken in den Hintergrund (Maasen, 2008), sodass mit zunehmender Akzeptanz der Schönheitschirurgie auch eine Normalisierung von Schönheit selbst stattfindet (unter Inkaufnahme der Stigmatisierung von jenen » nicht schönen « Personen, welche die auch zu Macht- und Herrschaftszwecken standardisierte Norm nicht erfüllen; vgl. Ruck, 2012). Normierte Schönheitskonzepte gibt es verschiedene (zum Beispiel das Modell des zeitlosen »year-zero face «; vgl. Borkenhagen \& Brähler, 2012). In populären Magazinen und anderen Medien sind heute körperoptimierende, ästhetische Terme und Praktiken präsent, die das längst konventionelle »Make- $\mathrm{Up}_{\mathrm{p}}$, den $\gg$ Beauty-Salon « und selbst »Botox « weit hinter sich lassen. »Ab-Crack «, »Contouring «, $\gg$ Detox «, $\gg$ Kryotherapie «, $\gg$ Microblading $\ll$ (als eine auch von Feministinnen wie Lena Dunham legitimierte mögliche Gegenmaßnahme gegen » feminine « Schönheitsideale, die Frauen zum Beispiel zur Ganzkörperenthaarung anhalten), »Post-BabyMakeover «, das »Thigh Gap« oder der »Undone-Look«, so lautet eine im ZEITMagazin vom 13. Juli 2017 auf Seite 18 erläuterte Liste aktueller Fachausdrücke, die sich auf einschlägige ästhetische Maßnahmen und Moden beziehen. Verschiedene Ideale und Verfahren konkurrieren um Hegemonie und die damit verwobenen Ressourcen (ökonomisches, soziales, kulturelles Kapital). Einige davon passen zusammen und fügen 
sich in ein übergeordnetes Konzept der Normierung und Normalisierung des »schönen Körpers «.

Weitere optimierende Interventionsmöglichkeiten bieten chronobiologische Prozesse, zum Beispiel der Schlaf. Dieser wurde insbesondere zu Beginn des 20. Jahrhunderts im Zuge der Elektrifizierung der Städte und vor allem der auf Effizienz bedachten Rationalisierung von Arbeitsprozessen zum Objekt umfangreicher Forschungsvorhaben und zahlreicher Optimierungsprogramme, da die Ermüdung und die Reproduktion der Arbeitskraft in den Arbeitswissenschaften im Kontext umfassender Industrialisierung zunehmend zu einem Problem wurden (Ahlheim, 2013). Insbesondere seit den 1950er Jahren entstand ein umfangreiches Potpourri an Schlafratgeberliteratur, die nicht nur einen optimalen, sondern auch den richtigen, da mit dem biologischen Rhythmus im Einklang stehenden Schlaf erlernbar machen sollte. Der heutige Schlaf wird zunehmend individueller, technisch vermittelter Überwachung und Kontrolle unterzogen: (Relativ) Erschwingliche Brustgurte, Armbanduhren sowie Hirnwellenmessgeräte, drahtlos verbunden mit einem mobilen Endgerät, versprechen anhand von akribischer Schlafdokumentation eine umfassende Analyse des Schlafverhaltens. Zwar sind geeignete technische Geräte erst seit Kurzem auf dem Markt. Doch die damit verbundenen Hoffnungen bleiben nach wie vor die Gleichen: kürzere Schlafzeiten bei gleichzeitiger Verbesserung der Schlafqualität und Steigerung der Produktivität (Meißner, 2016; Wimmer, 2015).

Programme der Körperoptimierung lassen sich nicht nur auf der Ebene des Eingriffs in die Integrität des Körpers verorten. Auch die Ergänzung oder Ersetzung des Körpers selbst - oder zumindest einzelner Teile - wird nach wie vor debattiert (Spreen, 2015, Straub \& Métraux, 2017). Auch diese (zunächst reparative, restaurative) Praxis ist nicht neu: Schon nach dem Ersten Weltkrieg wurde von diversen (auch staatlich alimentierten) Institutionen intensiv daran gearbeitet, Kriegsversehrte durch prothetische Apparaturen in die Gesellschaft zu reintegrieren (Harrasser, 2013). Ein wesentlicher Aspekt ist dabei ein spezifisches Verständnis des menschlichen Körpers als System diskreter, austauschbarer Teile, die (zum Beispiel den jeweiligen Anforderungen am Arbeitsplatz, aber auch individuellen Körpergefühlen, Nützlichkeitserwägungen und Schönheitsvorstellungen) entsprechend optimiert werden können. Spätestens mit der Einführung des Begriffs »Cybernetic Organism «, kurz: Cyborg, gewann dieser Gedanke kulturindustrielle Prominenz. Von Wissenschaftlern der NASA erdacht, (Clynes \& Kline, 1960) ging es alsbald um radikale technische Modifikationen und Supplemente des menschlichen Körpers, sodass dieser auch in den widrigsten und menschenfeindlichsten Umwelten des Weltalls »überlebt«. Das kybernetische Element bezeichnet hierbei eine optimale Kopplung zwischen bios und techné. Die Metapher des Cyborgs beziehungsweise der Cyborg ${ }^{4}$ wurde - ganz anders als in vielen Männerfantasien und patriarchalischen Wirklichkeiten - von Donna Haraway in ihrem berühmten Essay 
»Ein Manifest für Cyborgs « (1995, orig. 1984) für (und gegen) feministische Theorien entfaltet (Straub, 2018). Die Autorin argumentiert dort gegen Naturalismen, Essenzialismen und Universalismen, gegen Eindeutigkeiten und Festschreibungen im Namen einer starren, vielerlei Unterschiede ignorierenden und unterdrückenden Identitätspolitik. Haraway appelliert an unseren »Möglichkeitssinn «, wenn sie ihre Fantasie und Imagination mobilisiert, um für eine »Verschmelzung « von Differentem und Disparatem zu plädieren (das, wie das Paar »Mensch und Maschine « bis heute bezeugt, nicht ohne weiteres zusammengebracht werden konnte). Haraways Cyborg ist transgressiv: Sie überschreitet Grenzen wie die zwischen Natur und Kultur, Mensch und Maschine, Mann und Frau. Das Neue zeigt sich bei Harraway in der allerlei Grenzen, die bislang unantastbar schienen, überschreitenden Cyborg selbst. Dieses seltsame Mischwesen steht dabei pars pro toto: als Speerspitze exzessiven Enhancements symbolisiert die Cyborg das Ende aller eingeschliffenen Dualismen und (der damit verwobenen) Hierarchien. Haraways Verschmelzung von bios und techné, Natur und Kultur, Tier und Mensch, Mensch und Maschine ist emanzipatorisch gedacht. Nicht festgelegt sein zu wollen, Uneindeutigkeit zu affirmieren, anstatt sie zu beseitigen, markiert den politischen Aspekt und eigentlichen Kern von Haraways (nicht nur theoretischem) Plädoyer für die Cyborg. Diese metaphorische und zugleich reale Figur zeigt nicht zuletzt, wie radikales, exzessives Enhancement nicht nur auf die Verbesserung von Individuen und ihrer Körper zielt (und womöglich ihre Cyborgisierung in transgressiven TechnoPraxen betreibt), sondern ebenso auf den Umbau gesellschaftlicher Verhältnisse und sozialer Beziehungen. Psychisches ist dabei ebenfalls stets im Blick, auch wenn es augenscheinlich erst einmal um optimierende Modifikationen von biologischen Organismen und Techno-Körpern geht. Auch für diese genuin psycho-soziale Perspektive lassen sich zahlreiche Beispiele aus der Lebenspraxis und der wissenschaftlichen Forschung anführen.

Psychisches ist niemals vom Körper unabhängig (vice versa). Dies zeigt sich auch bei Praktiken der Selbstoptimierung, die darauf abzielen, ganz unmittelbar Aspekte der Seele zu ändern (Erleben, Verhalten, Kompetenzen oder Persönlichkeitseigenschaften). Dies wird zum Beispiel bei der Betrachtung von Neuroenhancements sehr deutlich. So sollen zum Beispiel durch die Einnahme von pharmakologischen Präparaten - die häufig zunächst zu therapeutischen Zwecken entwickelt wurden, um beispielsweise ADHS oder Narkolepsie zu behandeln - Aufmerksamkeits- und Aufnahmekapazitäten, Konzentrations- und Gedächtnisleistungen biochemisch verbessert werden, um kognitiv anspruchsvolle Aufgaben schneller und effizienter bearbeiten zu können (Crone, 2006; Müller, 2010; eine umfangreiche empirische Studie bietet Wagner, 2017). Auch Emotionen und komplexe Gefühlslagen können auf diesem Weg reguliert und optimiert werden (um zum Beispiel dem Ideal eines » ausgeglichenen Gemüts « oder » umfassender Affektkontrolle « näher zu kommen). 
Insbesondere aufgrund der ursprünglich kurativen Zwecksetzung einschlägiger Präparate - viele fallen in Deutschland unter das Betäubungsmittelgesetz und sind ohne ärztliches Rezept nicht frei verkäuflich - werden nicht allein Grenzen zwischen (ohnehin keineswegs disjunkten) Konzepten wie Gesundheit und Krankheit unklar (Lenk, 2011; grundsätzlich Schramme, 2012). In diesem Kontext wird auch der Status von psychischen Störungen (wie beispielsweise ADHS) selbst fragewürdig. Entsprechend wird diskutiert, ob durch die Diagnose von Störungen sozial deviantes Verhalten sozialen Normen angeglichen werden soll (Karsch, 2011; für eine empirische Untersuchung vgl. Shachak et al., 2013). Letztendlich stammt der Begriff des Enhancements, wie dargelegt, selbst aus der Medizinethik, wo die Abgrenzung von kurativen beziehungsweise therapeutischen und optimierenden Maßnahmen zentral ist (Talbot \& Wolf, 2006; Schöne-Seifert \& Talbot, 2009; Müller, 2010).

Diese medizinethische Diskussion lässt sich unter dem Begriff der $\gg$ therapeutischen Kultur « (Straub, 2013) in einen breiteren Kontext stellen. In den Fokus der Betrachtung geraten dabei nicht nur optimierende Praktiken im Kontext der Psychotherapie beziehungsweise Psychotechnik im Allgemeinen sowie psychologisch fundierte Beratungsangebote, sondern auch die sozialen, kulturellen und historischen Grundlagen solcher Praxen. Seit ca. einem halben Jahrhundert spricht man diesbezüglich (kritisch) von einer »therapeutischen Weltanschauung « in »Psy-Regimen «, in welcher das Wissenssystem der Psychologie und -therapie bereits zu Beginn des letzten Jahrhunderts zu einem allgemeinen, populärwissenschaftlichen und kulturellen Repertoire an Deutungs- und Verstehensangeboten (des Selbst, anderer Personen, sozialer Beziehungen) avancierte (z. B. Illouz, 2011; Rose, 1999, 2010; Tändler, 2016). Gleichzeitig konnte diese Weltanschauung nicht nur von Dienstleister_innen für die effektive Durchsetzung von Rationalisierungsprozessen und Produktivitätssteigerungen in Betrieben mobilisiert werden (Illouz, 2011), sondern es konnte sich auch ein umfassender Markt an Beratungsangeboten zwecks Optimierung des Familien- oder Sexuallebens oder auch allgemein zur Bewältigung von Stress etablieren (Maasen et al., 2011). Insbesondere bei der Betrachtung von psychologischen Strömungen wie der Humanistischen Psychologie wird deutlich, wie sehr die wissenschaftlich begründete und jeder Person verheißungsvoll anempfohlene Selbstaktualisierung und Selbstverwirklichung im Kontext einer » auteronomen « Struktur der therapeutischen Kultur zu verstehen ist, in der Einzelnen Selbstverantwortung und gleichzeitig Abhängigkeit qua Anleitung und Beratung vermittelt wird. Das ist längst ein zentraler Bestandteil eines weit gespannten Programms der Optimierung des Menschen (Straub, 2012c, 2013). Im Übrigen wurden in solchen (psychologisch, psychotherapeutisch, psychotechnisch geprägten) Zusammenhängen oftmals sogenannte weibliche Attribute und Eigenschaften (die Fähigkeit zum Zuhören und Kommunizieren, Empathie, Teamfähigkeit und andere soft skills) zu Wegmarken einer (vermeintlich) weltverbessernden Selbstoptimierung. 
Selbsthilfeliteratur zur Optimierung insbesondere der eigenen Produktivität finden sich heutzutage zuhauf. Die Literatur zum Zeit- und Aufgabenmanagement ist ebenso unüberschaubar wie das Angebot an Willens- und Motivationstrainings. Auf welche Art das Selbst angesprochen und mobilisiert und wie auf die individuelle psychische Konstitution zugegriffen werden soll, lässt sich exemplarisch an Werken verdeutlichen, die zwar aus der ersten Hälfte des letzten Jahrhunderts stammen, jedoch bis heute von ihrer Aktualität und Strahlkraft kaum etwas eingebüßt haben. Frühzeitig finden sich Techniken zur Steigerung der »Willenskraft « (exemplarisch: Broder Christiansens Ich will, ich kann! aus dem Jahr 1918; dazu Steinfeld, 2016; für einen historischen Überblick vgl. Cowan, 2013) und Programme zur umfassenden Selbstrationalisierung (exemplarisch: Gustav Großmanns Monografie Sich selbst rationalisieren von 1927). Schon in dieser Zeit gab es systematische Modi und detaillierte Anleitungen, um die gesamte Lebensführung von Personen, ihren Erfolg und ihr Glück, in Mittel-ZweckRelationen aufzugliedern. Das gilt bis heute als Königsweg, auf dem sich das von vielen erhoffte Gelingen einstellt, insofern die entsprechenden praktischen Anleitungen strikt befolgt und verfügbare Motivations- und Dokumentationstechniken genutzt werden (Rieger, 2002; Straub, 2017).

Für zahlreiche Optimierungshandlungen ist die (zu Beginn dieses Abschnitts bereits erwähnte) Veränderung des Sozialen der Ermöglichungsgrund oder ein gleichursprüngliches Phänomen wie die Verbesserung der Seele und des Körpers. Selbstoptimierungen lassen sich nur in einer Perspektive angemessen beschreiben und zufriedenstellend analysieren, in der soziokulturelle Bedingungen und Folgen mitberücksichtigt werden. Wegen zunehmender Dynamisierungen, Flexibilisierungen und Beschleunigungen der kapitalistischen Gesellschaft und Kultur (Rosa, 2012) wird es für viele nicht nur erforderlicher, sondern auch plausibler und generell akzeptabler, sich selbst als Gegenstand umfassender Verbesserungsmaßnahmen zu verstehen und zu behandeln. Wettbewerbsanforderungen, bedeutsame kulturelle Leitvorstellungen und beschleunigte Zeitregime verlangen es, dass Personen ihr Selbst und ihre Lebensführung rationalisieren, um weiterhin mithalten zu können. Dass diese Umstände auch Überforderung, Erschöpfung und Leid hervorrufen können, zeigen zahlreiche Studien (Ehrenberg, 2004, 2012). Jedoch müssen die Anpassungsleistungen kompetitiver, sich optimierender Subjekte keineswegs zwangsläufig in Destruktivität, Depression und andere Pathologien münden. Viele Menschen empfinden ihre Optimierungsbemühungen als gelungen und sind mit den Ergebnissen und Folgen zufrieden, genießen vielleicht narzisstische Gratifikationen und affirmieren schließlich auch Fremdzwänge so sehr, dass sie sich - zumindest zeitweise - wie eigene Motive » anfühlen « (Uhlendorf et al., 2016; King \& Gerisch, 2015; Schreiber et al., 2015; King, 2013a; King et al., 2014; Gerisch, 2013; King et al., 2017).

Auch die Lebensführung anderer Personen erscheint für viele im Lichte der Op- 
timierungskultur verbesserungswürdig. So zeigt sich auch in Studien zu Bildung und Erziehung, dass das pädagogische Handeln von Eltern unternehmerische Züge annehmen kann und Elternschaft, die Schulwahl sowie der Bildungsverlauf und die Freizeitgestaltung der Kinder in zweckrationalen Kosten-Nutzen-Analysen verhandelt und unter permanenten Optimierungsdruck gesetzt wird. Nicht allein am breiten Angebot von Elternratgebern ist dies zu beobachten (Thompson, 2015). Auch empirische Studien belegen, wie sehr Partnerschaft und Familienbeziehungen der effizienzorientierten Optimierungslogik einer beschleunigten Gesellschaft unterworfen werden und sich unversehens im Lebensalltag von Kindern und in den Orientierungen von Heranwachsenden niederschlagen können (King, 2013b).

Unsere exemplarische Liste von Handlungsfeldern und Lebensbereichen zeigt, dass beständige Optimierungen immer mehr zur allgemeinen Norm geworden sind (ohne dass dieser Vorgang der Generalisierung und auch der Radikalisierung unendlicher Weisen der Verbesserung des Selbst und der Welt abgeschlossen wäre). Der Körper, die Seele, die sozialen Beziehungen und kulturellen Lebensformen sind heute allesamt Gegenstände permanenter und mitunter exzessiver Optimierungen. Sowohl traditionelle Selbstformungen als auch innovative Enhancements gehören zum Alltag von Menschen, die unentwegt nach Verbesserungsoptionen und -programmen Ausschau halten, um als auteronome Subjekte selbst tätig zu werden oder sich von Expert_innen beraten und behandeln zu lassen. Aufregend und vielleicht besorgniserregend ist im 21. Jahrhundert insbesondere die Kultur eines schrankenlosen Enhancements, welche sich im äußersten Fall nicht mehr mit der Verbesserung des Menschen bescheidet, sondern die rastlos betriebene Optimierung als seine Abschaffung begreift. Auf diese programmatische Ambition, die heute eine überaus wichtige Schaltstelle einer technischen Kultur des Menschen-Machens bildet, möchten wir noch einen letzten Blick werfen. Dabei geht es nicht um kulturkonservative Technikkritik und Ressentiments gegen alles Neue. Romantische Verklärungen der verblassten Vergangenheit und schwindenden Gegenwart waren stets zweifelhaft und sind auch heute unangebracht. Eine auf weiten Strecken deskriptiv und analytisch verfahrende Sozialwissenschaft muss sich allerdings mit empirischen Befunden sowie theoretisch reflektierten Zeitdiagnosen nicht bescheiden. Zumal die Sozial- und Kulturpsychologie aufgefordert ist, auch Reflexionen anzustellen und anzuregen, die nicht gleich jede technische Transgression und Transformation des Menschen völlig vorbehaltlos als offenen Weg ins neue Glück begrüßen. Die psychologische Forschung ist wohl generell angehalten, die im vorliegenden Beitrag gesichteten Phänomene noch genauer zu erkunden, als es bislang geschehen ist. Dabei wird sie in jedem einzelnen Fall mögliche Licht- und Schattenseiten genau betrachten müssen, sogar dann, wenn es sich manchmal noch gar nicht um etablierte soziokulturelle und psychosoziale Wirklichkeiten handelt, sondern erst einmal um Imaginäres und Fiktives, von machtvollen Wünschen und starken Begehren angetriebene Vorstellungswelten, denen 
zumindest manche Menschen tatkräftig näherzukommen suchen (vgl. zu einem solchen Forschungsprogramm, das sich auch dem Noch-nicht-Wirklichen zuwendet und dabei des von Robert Musil geadelten »Möglichkeitssinns « der Forschenden bedarf, Straub, 2012b).

\section{Transgressives Human Enhancement der trans- und posthumanistischen Avantgarde}

Andreas Woyke (2010, S. 22f.) verweist zurecht auf Besonderheiten des Human Enhancement, wenn er Trans- und Posthumanisten vorwirft, gewichtige Differenzen zwischen diesem Typ der Optimierung und traditionellen Verbesserungen (oder auch therapeutischen Maßnahmen im Sinne der Restitution eines ehemaligen Zustandes beziehungsweise der Heilung oder Linderung von Leiden etc.) zu verwischen. Woyke betont, dass die wissenschaftlich-technisch avancierten Methoden des (insbesondere biologisch fundierten) Enhancement in der Tendenz auf eine regelrechte »Züchtung « künftiger Lebewesen im »Menschenpark « abzielen (Sloterdijk, 2001; dazu Straub et al., 2012). Diese neuen, künstlichen Geschöpfe werden, so heißt es, in bestimmten (oder sogar in so gut wie allen wichtigen) Hinsichten dem » antiquierten Menschen « haushoch überlegen sein (vgl. Sieben et al., 2012, darin speziell als Beispiel aus dem Feld der gentechnisch assistierten, eugenischen Menschenproduktion: Straub, 2012b; zum geflügelten Wort des » antiquierten Menschen« vgl. die bahnbrechende Studie von Anders, 1956, 1980). Insbesondere die sogenannten Lebenswissenschaften unserer Tage (life sciences wie die Biologie oder Medizin) korrigieren und verbessern nicht nur ein wenig, sondern steigern das Leben transgressiv, indem sie bisherige Grenzen sprengen und wirklich Neues hervorbringen. Manchmal streben sie sogar einen gänzlich »neuen Menschen « an, der dem alten so wenig ähnelt, dass ein anderer Name angebracht erscheint: der oder die Cyborg etwa. Bei den radikalen und exzessiven Varianten des naturwissenschaftlichen, technologisch und technisch ambitionierten Enhancement geht es nicht mehr um Verbesserungen des Althergebrachten, sondern um die Überwindung und Abschaffung des Menschen. Der sich optimierende Mensch hat sich in dieser utopischen - manche sagen: dystopischen - Perspektive schlicht überlebt, jedoch dabei selbst dafür gesorgt, dass sein Leben in transformierter Gestalt weitergehen kann. Die strukturellen (biologischen) Voraussetzungen bescheren dem neuen Lebewesen dabei eine funktionale Vollkommenheit, die, wie es manche Visionäre verheißen, im Modus der Unsterblichkeit vielleicht sogar unendlich andauern wird. Das kaum verborgene Vorbild dieser Vision ist die perfekte Maschine (Krüger, 2004, 2010).

Ganz unabhängig von der geläufigen Kritik an apologetischen Texten von Natur- 
beziehungsweise Neurowissenschaftler_innen (und sogar manchen philosophischen Legitimationsbemühungen von Bioethiker_innen) liegt es nahe, die im Betrieb der Lebenswissenschaften und Biotechnologien (sowie ihrer geschäftstüchtigen Vermarktung) häufiger ins Spiel kommenden Fortschrittsmythen und Vollkommenheitsphantasmen als Ausdruck narzisstischer Größen- und Allmachtsfantasien zu deuten. Die rhetorischsuggestiven Formeln, nach denen zunehmend (omni-)potente Menschen den »nicht würfelnden Gott « schon bald ablösen oder die nach Naturgesetzen verlaufende Evolution fortan selbst in die Hand nehmen werden, sind bezeichnende und selbstredende Zeugnisse für diese Allmachtsfantasien. Einschlägige Verlautbarungen, Programme und Projekte finden sich etwa bei Bostrom (2007), Bostrom und Roache (2007), Knoepffler und Savulescu (2009) oder Ranisch und Savulescu (2009). Müller (2010, S. 33ff.) kritisiert außerdem Texte von Henry Greely, Michael Gazzaniga und Martha J. Farah oder Thorsten Galert, Bettina Schöne-Seifert, Reinhard Merkel und Davinia Talbot, speziell deren »pseudo-ethische « Apologien des Human Enhancement und so gut wie aller Neuro-Enhancement-Präparate, indem er ihnen den Spiegel einer ancilla technologiae vorhält.

Fantasien einer tendenziell transgressiven Selbst-Steigerung entfalten schon in $\gg$ bescheideneren « Varianten der Selbstoptimierung ihre motivierende Kraft, etwa in starken Affirmationen technisch-apparativ vermittelter Weisen der Selbstquantifizierung, wie sie in der Quantified Self-Bewegung geschätzt und propagiert werden. Erschwingliche Messgeräte und Smartphone-Apps versprechen objektive Messungen unterschiedlichster Parameter (Schrittzahl, Kalorienhaushalt, Schlafqualität, Arbeitszeit etc.), um letztendlich $\gg$ Self-Knowledge through numbers « zu erlangen (Wolf, 2009). Ein universeller Anspruch kommt hier zum Tragen, geleitet von einem Ethos, in dem die technische Machbarkeit des »guten, gelingenden Lebens « mit der unhintergehbaren Objektivität des Messens in eins gesetzt wird (Balandis \& Straub, 2018b; Balandis, 2018; Duttweiler et al., 2016; Selke, 2016; Lupton, 2016; Neff \& Nafus, 2016). Gewiss ist nicht jede Messung im Zeichen einer möglichst umfassenden Selbst-Vermessung bereits ein alle alten Grenzen sprengender Schritt ins Leben eines » neuen Menschen «, vor dem antiquierte Ausgaben unausweichlich werden abdanken müssen. Jedoch können ein exakt vermessener Körper und eine quantifizierte Seele einer derartigen Transgression den Weg ebnen und Selbstoptimierungen zum radikalen, permanenten Exzess werden lassen. Psychologisch betrachtet ist dies außerordentlich wichtig, da auf diese Weise brauchbare Bereitschaften geweckt, schon vorhandene Motive gestärkt und bereits ansatzweise vorhandene Dispositionen gefestigt werden. Die auf Messungen abzielende Verdrahtung von Körper und Seele bildet eine wichtige Grundlage für Optimierungen, deren Richtung als Cyborgisierung zutreffend gekennzeichnet ist. Ob dabei (nicht nur der grammatikalischen Form nach) »männliche « Cyborgs (wie bei Bostrom, Savulesco und anderen Autoren, die vom unverletzlichen und unsterblichen, 
grenzenlos mächtigen Organismus träumen) oder aber »weibliche« Cyborgs (wie in Haraways radikal antinaturalistischem, antidogmatischem Plädoyer für die Uneindeutigkeit und Nicht-Festgelegtheit neuer Mischwesen) herauskommen, ist eine offene Frage.

Ebenso offen sind viele weitere Fragen, die einzelne Ergebnisse und Folgen technischer Maßnahmen der Optimierung des Selbst betreffen. Obwohl für verschiedene Phänomene (wie etwa Schönheitsoperationen, leistungssteigernde Medikamente oder die Auswirkungen von Gadgets und Apps, die zum Beispiel der Kontrolle und Verbesserung des Ernährungs-, Bewegungs- oder Schlafverhaltens dienen sollen) erste empirische Befunde vorliegen und neue Forschungsvorhaben angelaufen sind (zum Beispiel das von Vera King, Benigna Gerisch und Hartmut Rosa mit Unterstützung der Stiftung Volkswagenwerk seit 2017 unterstützte Projekt »Das vermessene Leben - Produktive und kontraproduktive Folgen der Quantifizierung in der digital optimierenden Gesellschaft «), kann von einer erschöpfenden Analyse der psychosozialen Aspekte vielfältigen Enhancements und traditioneller Maßnahmen der Selbstformung keine Rede sein. Wenn man dabei weitere Fortschritte erzielen will, ist man gut beraten, als Forscher_in eigene Vorlieben, Voreingenommenheiten und Vorurteile möglichst zurückzustellen. Nur dann lässt sich erkennen, welche Ergebnisse und Folgen die einzelnen Maßnahmen tatsächlich zeitigen - bei verschiedenen Individuen und Gruppen, die sich selbstverständlich in unterschiedlichen (Lebens-) Situationen befinden.

Im Übrigen lassen sich viele Phänomene nur mit dieser »gelassenen Einstellung « auch in ihrer hochgradigen Ambivalenz oder Polyvalenz wahrnehmen. In einem Feld, in dem die Grenze zwischen harmlosen Spielereien und folgenarmen Unterhaltungsritualen einerseits und dem (oft nur sehr allmählich verlaufenden) Eingriff in Subjektund Sozialformen andererseits nur sehr dünn sein kann, sollte man zunächst einmal genau sichten, was es so alles gibt und was dies für die Individuen oder Kollektive jeweils bedeutet. Dabei scheint eines von vorneherein klar: kaum jemand dürfte zu NeuroEnhancements greifen, wenn diese das eigene Selbst - der verheißungsvollen, versprochenen Wirkung zum Trotz - lediglich beeinträchtigen und (zumindest auf lange Sicht) beschädigen. Niemand verschreibt sich Programmen der Selbstoptimierung, wenn über kurz oder lang nur Schlechtes dabei herauskommt - und obendrein ohnehin nichts davon Spaß macht, Vergnügen bereitet, Lust verschafft und Befriedigung bietet. Menschen sind in der Regel keine autodestruktiven Akteure oder verkappte Masochisten (jedenfalls nicht vornehmlich). Sie tun, was sie tun, oftmals aus (subjektiv) guten Gründen und mit einer gewissen Freude an diesem so manche Wünsche und Sehnsüchte durchaus erfüllenden Tun. Technische beziehungsweise technisch vermittelte Formen der Selbstoptimierung sind oft Quellen der Beglückung und der Befriedigung vielfältiger Begehren und Wünsche. Selbstkontrolle, Selbstwirksamkeit, Selbststeigerung oder einfach das Erreichen kleinerer Ziele, die im niemals völlig ge- 
bändigten und geordneten Alltag gleichwohl wichtig sein können, gehören zu diesen Attraktoren und motivierenden Quellen - selbst wenn sie nicht die einzigen Effekte sind, die sich einstellen, wenn Menschen sich und ihre Welt zu optimieren gedenken. Das Fremdwerden des eigenen Körpers, die Ablenkung von eigentlich wichtigen Bestandteilen und Zwecken des eigenen Daseins, nicht zuletzt die mitunter unmerkliche Distanzierung von Anderen sowie eine gewisse Vereinzelung sich selbst verbessernder Individuen können die angestrebten und willkommenen Wirkungen der jeweils ergriffenen (oder erduldeten) Maßnahmen begleiten und diese sogar untergraben und nichtig machen (Balandis \& Straub, 2018; Straub \& Balandis 2018). Man kann wohl festhalten: Ambivalenz ist nicht unbedingt ein Merkmal des Neuro-Enhancement und anderer Modi der Selbstverbesserung, das zu allzu großer Sorge Anlass gibt. Besorgniserregend wird es erst, wenn die Mehr- und Vieldeutigkeit verschwindet und sich vermeintliche Verbesserungen als falsche Versprechen und bloßer Schwindel erweisen, dem zu Illusionen neigende, auf (möglichst technisch vermittelte, möglichst präzise) Kontrollen des eigenen Lebens versessene Selbstoptimierer auf den Leim gegangen sind.

\section{Anmerkungen}

1 Zum Folgenden vgl. ausführlicher Straub et al. (2012b). Wir übernehmen hier ein paar Formulierungen aus diesem Text, in dem sich neben terminologischen Reflexionen auch theoretische Systematisierungsvorschläge sowie empirische Beispiele aus dem heterogenen Feld des optimierenden »Menschen-Machens« finden. Die Implizität von (unter Umständen durchaus vagen, keineswegs exakt metrisierbaren äußeren und obendrein instabilen, flexiblen) Kriterien der optimierenden Selbstveränderung besagt übrigens, dass sich die Subjekte keineswegs völlig darüber im Klaren sein müssen, in welche Richtung sie sich bei ihren (polyvalenten) Optimierungsbemühungen eigentlich bewegen.

2 Dieses »Wissen« muss keine gesicherte Erkenntnis im Sinne wissenschaftlicher Theorien und Befunde sein. Das Alltagswissen kommt ebenso in Betracht wie sonstige (z. B. religiöse, ästhetische) Überzeugungssysteme, die zwischen (allgemein anerkanntem) Wissen, (subjektiv evidentem) Glauben und (bloßem) Meinen changieren mögen oder ganz einem dieser drei Wissenstypen zuzurechnen sind.

3 Einige Formulierungen in diesem Abschnitt finden sich (in kürzerer Fassung) auch in einem Beitrag im Handbuch interdisziplinäre Geschlechterforschung (Balandis \& Straub, 2018a), wobei dort häufiger geschlechtsspezifische Aspekte akzentuiert werden (die auch hier nicht völlig unberücksichtigt bleiben dürfen, weil sie viele Ideale, Praktiken und Methoden der Optimierung strukturieren und manchmal sogar konstituieren). Ohne (relativ) verfestigte Geschlechterdifferenzen und Geschlechterbeziehungen in patriarchalen Ungleichheitsverhältnissen verlören manche Weisen der Selbstoptimierung und speziell des Enhancements zumindest einen Teil ihrer Bedeutung, ihres Sinnes und ihrer Funktion.

4 Haraway spricht ausdrücklich von der Cyborg und unterscheidet dieses Konzept vom ,männlich' konnotierten Cyborg. 


\section{Literatur}

Ach, J.S. (2006). Komplizen der Schönheit? Anmerkungen zur Debatte über die ästhetische Chirurgie. In J. Ach \& A. Pollmann (Hrsg.), no body is perfect. Baumaßnahmen am menschlichen Körper. Bioethische und ästhetische Aufrisse (S. 187-206). Bielefeld: transcript.

Ahlheim, H. (2013). Die Vermessung des Schlafs und die Optimierung des Menschen. Eine deutschamerikanische Geschichte, 1930-1960. Zeithistorische Forschungen/Studies in Contemporary History, 13, 13-37.

Anders, G. (1956). Die Antiquiertheit des Menschen. Band I: Über die Seele im Zeitalter der zweiten industriellen Revolution. München: Beck.

Anders, G. (1980). Die Antiquiertheit des Menschen. Band II: Über die Zerstörung des Lebens im Zeitalter der dritten industriellen Revolution. München: Beck.

Assmann, A. \& Assmann, J. (Hrsg.). (2010). Vollkommenheit. München: Wilhelm Fink.

Balandis, O. (2018). Selbstvermessung wider Willen. Eine Fallanalyse zu psychosozialen Aspekten technisch vermittelter Selbstoptimierung in der gegenwärtigen Technikkultur. psychosozial, 41(2), 74-95.

Balandis, O. \& Straub, J. (2018a). Selbstoptimierung und Enhancement. Begriffe, Befunde und Perspektiven für die Geschlechterforschung. In B. Kortendiek, B. Riegraf \& K. Sabisch (Hrsg.), Handbuch interdisziplinäre Geschlechterforschung. https://doi.org/10.1007/978-3-658-12500-4_81-1 (Stand: 27.02.2018).

Balandis, O. \& Straub, J. (2018). Self-Tracking als technische Selbstvermessung im Zeichen der Optimierung. Vom Nerd zum Normalverbraucher. Einführung in den Themenschwerpunkt. Das sich vermessende Selbst. psychosozial, 41(2), 5-15.

Borkenhagen, A. \& Brähler, E. (2012). Simply to look done. Das neue zeitlose Gesicht der Schönheitschirurgie. In A. Borkenhagen \& E. Brähler (Hrsg.), Die Selbstverbesserung des Menschen. Wunschmedizin und Enhancement aus medizinpsychologischer Perspektive (S. 179-188). Gießen: Psychosozial-Verlag.

Borkenhagen, A., Brinkschulte, E., Frommer, J. \& Brähler, E. (Hrsg.). (2016). Schönheitsmedizin. Kulturgeschichte, ethische und medizinpsychologische Perspektiven. Gießen: Psychosozial-Verlag.

Bostrom, N. (2007). Dignity and Enhancement. http://tinyurl.com/nbostred (Stand: 01.02.2018).

Bostrom, N. \& Roache, R. (2007). Ethical Issues in Human Enhancement. http://www.nickbostrom.com/ ethics/human-enhancement.pdf (Stand: 26.05.2018)

Bröckling, U. (2007). Das unternehmerische Selbst. Soziologie einer Subjektivierungsform. Frankfurt/M.: Suhrkamp.

Christiansen, B. (als Uve Jens Kruse). (1918). Ich will - ich kann! Eine Schule des Willens und der Persönlichkeit. Buchenbach in Baden: Felsen Verlag.

Clynes, M. E. \& Kline, N. S. (1960). Cyborgs and Space. Astronautics September, 26-27 \& 74-76.

Coenen, C., Gammel, S., Heil, R. \& Woyke, A. (Hrsg.). (2010). Die Debatte über »Human Enhancement«. Historische, philosophische und ethische Aspekte der technologischen Verbesserung des Menschen. Bielefeld: transcript.

Cowan, M. (2013). Energieregulierung. Willenskultur und Willenstraining um 1900. In B. Gronau (Hrsg.). Szenarien der Energie. Zur Ästhetik und Wissenschaft des Immateriellen (S. 87-108). Bielefeld: transcript.

Crone, K. (2006). Gedächtnispillen. Mögliche Auswirkungen auf das Selbstverhältnis von Personen. In J. Ach \& A. Pollmann (Hrsg.), no body is perfect. Baumaßnahmen am menschlichen Körper. Bioethische und ästhetische Aufrisse (S. 233-252). Bielefeld: transcript.

Degele, N. (2004). Sich schön machen. Zur Soziologie von Geschlecht und Schönheitshandeln. Wiesbaden: VS Verlag für Sozialwissenschaften. 
Degele, N. (2008). Normale Exklusivitäten. Schönheitshandeln, Schmerznormalisieren, Körper inszenieren. In P.-I.Villa (Hrsg.), schön normal. Manipulationen am Körper als Technologien des Selbst (S. 67-84). Bielefeld: transcript.

Duttweiler, S. (2003). Body-Consciousness. Fitness - Wellness - Körpertechnologien des Selbst. Widersprüche. Zeitschrift für sozialistische Politikim Bildungs-, Gesundheits- und Sozialbereich. Selbsttechnologien, Heft 87(Technologien des Selbst). http://www.widersprueche-zeitschrift.de/article1027. html (Stand: 01.02.2018).

Duttweiler, S. (2012). Körper - Gesundheit - Sport. Selbsttechnologien in der Gesundheits- und Sportgesellschaft. Sozialwissenschaften und Berufspraxis, 35, 5-19.

Duttweiler, S., Gugutzer, R., Strübing, J., Passoth, J.-H. (Hrsg.). (2016). Leben nach Zahlen. Self-Tracking als Optimierungsprojekt? Bielefeld: transcript.

Ehrenberg, A. (2004). Das erschöpfte Selbst. Frankfurt/M.: Campus.

Ehrenberg, A. (2012). Das Unbehagen in der Gesellschaft. Berlin: Suhrkamp.

Foucault, M. (2006). Die Geburt der Biopolitik. Geschichte der Gouvernementalität II. Vorlesungen am Collège de France 1978/1979. Frankfurt/M.: Suhrkamp.

Gehring, P. (2006). Was ist Biomacht? Vom zweifelhaften Mehrwert des Lebens. Frankfurt/M.: Campus.

Gerisch, B. (2013). Von jagender Hast und vorzeitigem Zusammenbruch. Zur Psychodynamik schleichender Veränderungen in beschleunigten Zeiten. Journal für Psychoanalyse, 54, 7-28.

Gordijn, B. \& Chadwick, R. (Hrsg.). (2008). Medical Enhancement and Posthumanity. Berlin, Heidelberg: Springer.

Großmann, G. (1993 [1927]). Sich selbst rationalisieren: Lebenserfolg ist erlernbar. Vorbereitende Einführung in den elementaren Teil der Großmann-Methode. Grünwald: Ratio-Verlag.

Haraway, D. (1995 [1984]). Ein Manifest für Cyborgs. Feminismus im Streit mit den Technowissenschaften. In D. Haraway, Die Neuerfindung der Natur. Primaten, Cyborgs und Frauen (S. 33-77). Frankfurt/M., New York: Campus Verlag.

Harrasser, K. (2013). Körper 2.0. Über die technische Erweiterbarkeit des Menschen. Bielefeld: transcript.

Illouz, E. (2007). Gefühle in Zeiten des Kapitalismus. Frankfurter Adorno-Vorlesungen 2004. Frankfurt/M.: Suhrkamp.

Illouz, E. (2011). Die Errettung der modernen Seele. Therapien, Gefühle und die Kultur der Selbsthilfe. Frankfurt/M.: Suhrkamp.

Juengst, E.T. (1998). What Does Enhancement Mean? In E. Parens (Hrsg.), Enhancing Human Traits: Ethical and Social Implications (S. 29-47). Washington: Georgetown University Press.

Karsch, F. (2011). Neuro-Enhancement oder Krankheitsbehandlung? Zur Problematik der Entgrenzung von Krankheit und Gesundheit am Beispiel ADHS. In W. Viehöver \& P. Wehling (Hrsg.), Entgrenzung der Medizin. Von der Heilkunst zur Verbesserung des Menschen? (S. 121-142). Bielefeld: transcript.

King, V. (2013a). Die Macht der Dringlichkeit. Kultureller Wandel von Zeitgestaltungen und psychischen Verarbeitungsmustern. Schweizer Archiv für Neurologie und Psychiatrie, 164, 223-231.

King, V. (2013b). Optimierte Kindheiten. In F. Dammasch \& M. Teising (Hrsg.), Das modernisierte Kind (S. 31-51). Frankfurt/M.: Brandes \& Apel.

King, V. \& Gerisch, B. (2015). Perfektionierung und Destruktivität - eine Einführung. psychosozial, 38(3), $5-12$.

King, V., Gerisch, B. \& Rosa, H. (Hrsg.). (2017). »Lost in Perfection«. Impacts of Optimisation on Culture and Psyche. London: Routledge.

King, V., Lindner, D., Schreiber, J., Busch, K., Uhlendorf, N., Beerbom, C., Salfeld-Nebgen, B., Gerisch, B. \& Rosa, H. (2014). Optimierte Lebensführung - wie und warum sich Individuen den Druck zur Selbstverbesserung zu eigen machen. Jahrbuch für Pädagogik: Menschenverbesserung - Transhumanismus (29), 283-299. 
Kipke, R. (2011). Besser werden. Eine ethische Untersuchung zu Selbstformung und Neuro-Enhancement. Paderborn: Mentis.

Kipke, R. (2012). Ignoriert, dementiert, kritisiert: menschliche Selbstformung im Schatten der technischen Optimierungsstrategien. In A. Sieben, K. Sabisch-Fechtelpeter \& J. Straub (Hrsg.), Menschen machen. Die hellen und dunklen Seiten humanwissenschaftlicher Optimierungsprogramme (S. 269-303). Bielefeld: transcript.

Knoepffler, N. \& Savulescu, J. (Hrsg.). (2009). Derneue Mensch? Enhancement und Genetik. Freiburg, München: Alber.

Krüger, O. (2004). Virtualität und Unsterblichkeit. Die Visionen des Posthumanismus. Freiburg: Rombach.

Krüger, O. (2010). L'homme machine oder die Überwindung und Vervollkommnung des Menschen im Posthumanismus. In A. Assmann, \& J. Assmann (Hrsg.), Vollkommenheit (S. 107-129). München: Fink.

Kurzweil, R. \& Grossman, T. (2005). Fantastic Voyage: Live Long Enough to Live Forever. New York: Plume.

Lenk, Christian (2006). Verbesserung als Selbstzweck? Psyche und Körper zwischen Abweichung, Norm und Optimum. In J. Ach, \& A. Pollmann (Hrsg.), no body is perfect. Baumaßnahmen am menschlichen Körper. Bioethische und ästhetische Aufrisse (S. 63-78). Bielefeld: transcript.

Lenk, C. (2011). Enhancement vor dem Hintergrund verschiedener Konzepte von Gesundheit und Krankheit. In W. Viehöver \& P. Wehling (Hrsg.), Entgrenzung der Medizin. Von der Heilkunst zur Verbesserung des Menschen? (S. 67-88). Bielefeld: transcript.

Lupton, D. (2016). The Quantified Self: A Sociology of Self-Tracking. Malden: Polity Press.

Maasen, S. (2008). Bio-ästhetische Gouvernementalität. Schönheitschirurgie als Biopolitik. In P.-I. Villa (Hrsg.), schön normal. Manipulationen am Körper als Technologien des Selbst (S. 99-118). Bielefeld: transcript.

Maasen, S., Elberfeld, J., Eitler, P. \& Tändler, M. (Hrsg.). (2011). Das beratene Selbst. Zur Genealogie der Therapeutisierung in den slangen siebzigern. Bielefeld: transcript.

Mayer, R., Thompson, C. \& Wimmer, M. (2013). Inszenierungen und Optimierungen des Selbst. Zur Analyse gegenwärtiger Selbsttechnologien. Wiesbaden: VS Verlag für Sozialwissenschaften.

Meißner, Stefan (2016). Der vermessene Schlaf. Quantified Self in der Spannung von Disziplinierung und Emanzipation. In S. Duttweiler, R. Gugutzer, J.-H. Passoth \& J. Strübing (Hrsg.), Leben nach Zahlen. Self-Tracking als Optimierungsprojekt? (S. 325-347). Bielefeld: transcript.

Meyer-Drawe, K. (2008). Diskurse des Lernens. München: Fink.

Müller, O. (2010). Zwischen Mensch und Maschine. Vom Glück und Unglück des Homo faber. Frankfurt/M.: Suhrkamp.

Neff, G. \& Nafus, D. (2016). Self-Tracking. Cambridge: The MIT Press.

Neswald, E. R. (2013). Kapitalistische Kalorien. Energie und Ernährungsökonomie um die Jahrhundertwende. In B. Gronau (Hrsg.), Szenarien der Energie. Zur Ästhetik und Wissenschaft des Immateriellen (S. 87-108). Bielefeld: transcript.

Ranisch, R. \& Savulescu, J. (2009). Ethik und Enhancement. In N. Knoepffler \& J. Savulescu (Hrsg.), Der neue Mensch? Enhancement und Genetik (S. 21-53). Freiburg, München: Alber.

Reichenbach, R. (2009). Soft skills: destruktive Potentiale des Kompetenzdenkens. In C. Rohlfs, M. Harring \& C. Palentien (Hrsg.), Kompetenz-Bildung. Soziale, emotionale und kommunikative Kompetenzen von Kindern und Jugendlichen. Wiesbaden: VS Verlag für Sozialwissenschaften.

Rieger, S. (2002). Arbeit an sich. Dispositive der Selbstsorge in der Moderne. In U. Bröckling \& E. Horn (Hrsg.), Anthropologie der Arbeit (S. 79-96). Tübingen: Günter Narr Verlag.

Rosa, H. (2012). Weltbeziehungen im Zeitalter der Beschleunigung. Frankfurt/M.: Suhrkamp.

Rose, N. (1999). Governing the Soul. The Shaping of the Private Self. London: Free Association Books.

Rose, N. (2010). Inventing our Selves: Psychology, Power, and Personhood. Cambridge: Cambridge University Press. 
Rosen, C. (2004). The Democratization of Beauty. The New Atlantis. A Journal of Technology and Society, 5, 19-35.

Ruck, N. (2012). Zur Normalisierung von Schönheit und Schönheitschirurgie. In A. Sieben, K. SabischFechtelpeter \& J. Straub (Hrsg.), Menschen machen: Die hellen und die dunklen Seiten humanwissenschaftlicher Optimierungsprogramme (S. 79-105). Bielefeld: transcript.

Schmidt-Semisch, H. \& Schorb, F. (Hrsg.). (2008). Kreuzzug gegen Fette. Sozialwissenschaftliche Aspekte des gesellschaftlichen Umgangs mit Übergewicht und Adipositas. Wiesbaden: VS Verlag für Sozialwissenschaften.

Schöne-Seifert, B. \& Talbot, D. (Hrsg.). (2009). Enhancement. Die ethische Debatte. Paderborn: mentis Verlag.

Schramme, T. (Hrsg.). (2012). Krankheitstheorien. Frankfurt/M.: Suhrkamp.

Schreiber, J., Uhlendorf, N., Lindner, D., Gerisch, B., King, V. \& Rosa, H. (2015). Optimierung zwischen Zwang und Zustimmung. Institutionelle Anforderungen und psychische Bewältigungen im Berufsleben. Psychosozial 38(3), 27-42.

Selke, S. (Hrsg.). (2016). Lifelogging. Digitale Selbstvermessung und Lebensprotokollierung zwischen disruptiver Technologie und kulturellem Wandel. Wiesbaden: VS Verlag für Sozialwissenschaften.

Shachak, M., Díaz, E.C., Cohen, M.Á. \& Illouz, E. (2013). Psychiatry as Culture. Transforming childhood through ADHD. In M. Dellwig \& M. Harbusch (Hrsg.), Krankheitskonstruktionen und Krankheitsbrei. Die Renaissance der soziologischen Psychiatriekritik (S. 75-102). Wiesbaden: VS Verlag für Sozialwissenschaften.

Sieben, A., Sabisch-Fechtelpeter, K. \& Straub, J. (Hrsg.). (2012). Menschen machen. Die hellen und dunklen Seiten humanwissenschaftlicher Optimierungsprogramme. Bielefeld: transcript.

Sloterdijk, P. (2001). Regeln für den Menschenpark. Ein Antwortschreiben zu Heideggers Brief über den Humanismus. In P. Sloterdijk, Nicht gerettet. Versuche nach Heidegger (S. 302-337). Frankfurt/M.: Suhrkamp.

Sloterdijk, P. (2009). Du mußt dein Leben ändern. Über Anthropotechnik. Frankfurt/M.: Suhrkamp.

Spreen, D. (2015). Upgradekultur. Der Körper in der Enhancementgesellschaft. Bielefeld: transcript.

Steinfeld, T. (2016). Ich will, ich kann. Moderne und Selbstoptimierung. Konstanz: Konstanz University Press.

Straub, J. (2007). Kompetenz. In J. Straub, A. Weidemann \& D. Weidemann (Hrsg.), Handbuch Interkulturelle Kommunikation und Interkulturelle Kompetenz (S. 35-46). Stuttgart: Metzler.

Straub, J. (2012a). Optimierungstypen. Ein provisorisches Nachwort zu Licht- und Schattenseiten von Menschenverbesserungsprogrammen. In A. Sieben, K. Sabisch-Fechtelpeter \& J. Straub (Hrsg.), Menschen machen. Die hellen und dunklen Seiten humanwissenschaftlicher Optimierungsprogramme (S. 473-490). Bielefeld: transcript.

Straub, J. (2012b). Der naturalisierte und der programmierte Mensch. Lebenswissenschaften, Bioethik und psychosozialer Wandel: Psychologische Annotationen zu Jürgen Habermas' Sorge um eine optimierende Eugenik. In A. Sieben, K. Sabisch-Fechtelpeter \& J. Straub (Hrsg.), Menschen machen. Die hellen und dunklen Seiten humanwissenschaftlicher Optimierungsprogramme (S. 107-142). Bielefeld: transcript.

Straub, J. (Hrsg.). (2012c). Der sich selbst verwirklichende Mensch. Über den Humanismus der humanistischen Psychologie. Bielefeld: transcript.

Straub, J. (2013). Selbstoptimierung im Zeichen der »Auteronomie«. Paradoxe Strukturen der normierten Selbststeigerung. Von der »therapeutischen Kultur« zur »Optimierungskultur«. Psychotherapie \& Sozialwissenschaft, 2, 5-38.

Straub, J. (2017). Rationalising Life by Means of Self-Optimisation. In V. King, B. Gerisch \& H. Rosa (Hrsg.), "Lost in Perfection«. Impacts of Optimisation on Culture and Psyche (S. 153-188). London: Routledge. 
Straub, J. (2018). Vom Prothesengott zur Psychoprothese. Über prothetische Therapien und Optimierungen des Selbst in Psy-Regimen. (Drucklegung in Vorbereitung)

Straub, J. \& Balandis, O. (2018). Niemals genug! Selbstoptimierung und Enhancement als attraktive und riskante Praktiken verbesserungswilliger Menschen. Familiendynamik, 43(1), 72-82.

Straub, J. \& Métraux, A. (Hrsg.). (2017). Prothetische Transformationen des Menschen: Ersatz, Ergänzung, Erweiterung, Ersetzung. Bochum: Westdeutscher Universitätsverlag.

Straub, J., Sabisch-Fechtelpeter, K. \& Sieben, A. (2012a). Homo modificans, homo modificatus. Ein Vorwort zu aktuellen »Optimierungen des Menschen«. In J. Straub, K. Sabisch-Fechtelpeter \& A. Sieben (Hrsg.), Menschen machen. Die hellen und dunklen Seiten humanwissenschaftlicher Optimierungsprogramme (S. 9-26). Bielefeld: transcript.

Straub, J., Sieben, A. \& Sabisch-Fechtelpeter, K. (2012b). Menschen besser machen. Terminologische und theoretische Aspekte vielgestaltiger Optimierungen des Humanen. In J. Straub, K. SabischFechtelpeter \& A. Sieben (Hrsg.), Menschen machen. Die hellen und dunklen Seiten humanwissenschaftlicher Optimierungsprogramme (S. 27-78). Bielefeld: transcript.

Talbot, D. \& Wolf, J. (2006). Dem Gehirn auf die Sprünge helfen. Eine ethische Betrachtung zur Steigerung kognitiver und emotionaler Fähigkeiten durch Neuro-Enhancement. In J. Ach \& A. Pollmann (Hrsg.), no body is perfect. Baumaßnahmen am menschlichen Körper. Bioethische und ästhetische Aufrisse (S. 187-206). Bielefeld: transcript.

Tändler, M. (2016). Das therapeutische Jahrzehnt. Der Psychoboom in den siebziger Jahren. Göttingen: Wallstein.

Thompson, C. (2015). Eltern als >Bildungsunternehmer«. Zur Ausweitung und Radikalisierung optimiertoptimierender Bildung. psychosozial, 38(3), 27-42.

Tirosh-Samuelson, H. \& Mossman K. L. (Hrsg.). (2012). Building Better Humans? Refocusing the Debate on Transhumanism. Frankfurt/M.: Peter Lang.

Uhlendorf, N., Schreiber, J., King, V., Gerisch, B., Rosa, H. \& Busch, K. (2016). »|mmer so dieses Gefühl, nicht gut genug zu sein«. Optimierung und Leiden. Psychoanalyse im Widerspruch, 54(2), 31-50.

Villa, P.-I. (2011). Mach mich schön! Geschlecht und Körper als Rohstoff. In W. Viehöver \& P. Wehling (Hrsg.), Entgrenzung der Medizin. Von der Heilkunst zur Verbesserung des Menschen? (S. 143-162). Bielefeld: transcript.

Villa, P.-I. (2013). Rohstoffisierung. Zur De-Ontologisierung des Geschlechtskörpers. In R. John, J. RückertJohn \& E. Esposito (Hrsg.), Ontologien der Moderne (S. 225-240). Wiesbaden: VS Verlag für Sozialwissenschaften.

Villa, P.-I. \& Zimmermann, K. (2013). Fitte Frauen - Dicke Monster? Empirische Exploration zu einem Diskurs von Gewicht. In H. Schmidt-Semisch \& F. Schorb (Hrsg.), Kreuzzug gegen Fette. Sozialwissenschaftliche Aspekte des gesellschaftlichen Umgangs mit Übergewicht und Adipositas (S. 171-190). Wiesbaden: VS Verlag für Sozialwissenschaften.

Volkers, A. \& Lemke, T. (Hrsg.). (2014). Biopolitik. Ein Reader. Berlin: Suhrkamp.

Wagner, G. (2017). Selbstoptimierung. Praxis und Kritikvon Neuroenhancement. Frankfurt/M.: Campus.

Wiedemann, L. (2016). Vom Piksen zum Scannen, vom Wert zu Daten. Digitalisierte Selbstvermessung im Kontext Diabetes. In S. Duttweiler, R. Gugutzer, J.-H. Passoth \& J. Strübing (Hrsg.), Leben nach Zahlen. Self-Tracking als Optimierungsprojekt? (S. 293-324). Bielefeld: transcript.

Wimmer, M. (2015). Perfektionierung des Unverbesserlichen: unvermeidbar und unmöglich. psychosozial, 38(3), 81-99.

Wolf, G. (2009). Know Thyself. Tracking Every Facet of Life, From Sleep to Mood, to Pain, 24/7/365. https://www.wired.com/2009/06/lbnp-knowthyself/ (Stand: 07.12.17).

Woyke, A. (2010). Human Enhancement und seine Bewertung - eine kleine Skizze. In C. Coenen, S. Gammel, R. Heil, \& A. Woyke (Hrsg.), Die Debatte über»Human Enhancement«. Historische, philosophische und ethische Aspekte der technologischen Verbesserung des Menschen (S. 21-38). Bielefeld: transcript. 
Zillien, N. Fröhlich, G. \& Kofahl, D. (2016). Ernährungsbezogene Selbstvermessung. Von der Diätetik bis zum Diet Tracking. In S. Duttweiler, R. Gugutzer, J. Strübing \& J.-H. Passoth (Hrsg.), Leben nach Zahlen. Self-Tracking als Optimierungsprojekt? (S. 123-140). Bielefeld: transcript.

ZEIT-Magazin (2017). Strahlend schön. 72 Jg., Nr. 29, 13. Juli 2017. Hamburg: Zeitverlag Gerd Bucerius.

\section{Die Autoren}

Oswald Balandis, M. A., Jg. 1990, promoviert zu technisch vermittelter Subjektivierung und Praktiken der Selbstoptimierung. Seit 2016 ist er wissenschaftlicher Mitarbeiter am Lehrstuhl für Sozialpsychologie und Sozialtheorie an der Fakultät für Sozialwissenschaft der Ruhr-Universität Bochum.

Kontakt: E-Mail: Oswald.Balandis@rub.de

Jürgen Straub, Prof. Dr. phil., Dipl.-Psych., Jg. 1958, Promotion 1989, Habilitation 1995; 1994-95 Fellow am Zentrum für interdisziplinäre Forschung der Universität Bielefeld,1999-2002 Fellow am Kulturwissenschaftlichen Institut Essen, 2010 MERCURFellowship (Stiftung Mercator), 2016-17 Distinguished Robert Bellah-Fellowship (der Max-Planck-Gesellschaft) am Max Weber-Kolleg für kultur- und sozialwissenschaftliche Studien der Universität Erfurt. 2015 erhielt er den Ernst-Eduard-Boesch-Preis für Kulturpsychologie der Gesellschaft für Kulturpsychologie in Salzburg, 2017 den Höffmann-Wissenschaftspreis für Interkulturelle Kompetenz der Universität Vechta. Er ist seit 2008 Inhaber des Lehrstuhls für Sozialtheorie und Sozialpsychologie in der Fakultät für Sozialwissenschaft der Ruhr-Universität Bochum, von 2010 bis 2013 war er Dekan ebendort; seit 2015 ist er Kodirektor (mit Pradeep Chakkarath) des Hans Kilian und Lotte Köhler-Centrums für sozial- und kulturwissenschaftliche Psychologie und historische Anthropologie (KKC).

Kontakt: E-Mail: Juergen.Straub@rub.de 